Archives de sciences sociales des religions

127 | juillet - septembre 2004

Max Weber, la religion et la construction du social

\title{
Rationalisation et pluralité des rationalités
}

Gérard Raulet

\section{OpenEdition}

Journals

Édition électronique

URL : http://journals.openedition.org/assr/1053

DOI : 10.4000/assr. 1053

ISSN : $1777-5825$

Éditeur

Éditions de l'EHESS

Édition imprimée

Date de publication : 1 juillet 2004

Pagination : 79-91

ISBN : 2-222-96751-1

ISSN : 0335-5985

Référence électronique

Gérard Raulet, «Rationalisation et pluralité des rationalités », Archives de sciences sociales des religions [En ligne], 127 | juillet - septembre 2004, mis en ligne le 25 juin 2007, consulté le 19 avril 2019. URL http://journals.openedition.org/assr/1053; DOI : 10.4000/assr.1053 
Arch. de Sc. soc. des Rel., 2004, 127, (juillet-septembre 2004) 79-91

Gérard RAULET

\section{RATIONALISATION ET PLURALITÉ DES RATIONALITÉS}

\section{Religion et rationalisation : le refus wébérien de toute explication univoque}

On sait que Max Weber récuse avec une certaine virulence la thèse selon laquelle la religion et l'éthique religieuse ne seraient que des manifestations des rapports sociaux et de la situation sociale de leurs adeptes. Ce contre quoi il se dresse est un marxisme vulgaire pour lequel «les 'idées' sont le 'reflet' ou la 'superstructure' de situations économiques qui les génèrent» (1). Il fait remarquer qu'en Nouvelle Angleterre " 'l'esprit capitaliste' [était sans nul doute apparu] avant le 'développement du capitalisme' » et que "dans ce cas précis le rapport de cause à effet est donc exactement inverse à celui qu'on devrait postuler d'un point de vue 'matérialiste'»(2). En soulignant "dans ce cas précis» il signale toutefois qu'il entend pour sa part se garder de toute généralisation précipitée. De fait, au fil de son étude, il se «contentera» d'accumuler les exemples extrêmes qui parlent contre une application mécanique du schéma causaliste base - superstructure. Par exemple :

« Comment expliquer historiquement qu'à Florence, la ville qui était au centre du développement capitaliste de l'époque et fut, aux $\mathrm{XIV}^{\mathrm{e}}$ et $\mathrm{XV}^{\mathrm{e}}$ siècles, le marché d'argent et de capitaux de toutes les grandes puissances politiques, on ait tenu pour moralement condamnable ou pour seulement tolérable ce qui, dans les conditions mesquines et provinciales de la Pennsylvanie du XVIII ${ }^{\mathrm{e}}$ siècle, où l'économie menaçait constamment de sombrer dans l'échange naturel par pur manque de liquidités, où il y avait à peine trace

(1) Die Protestantische Ethik und der Geist des Kapitalismus est citée selon l'édition de Johannes Winckelmann : Die protestantische Ethik, Gütersloh, Gütersloher Verlagshaus G. Mohn, 1991 (8e édition), sous la forme suivante : PE et numéro de page (ici : PE 46) ; la traduction française utilisée est celle récemment proposée par Isabelle KALINOWSKI : Max Weber, L'éthique protestante et l'esprit du capitalisme, Paris, Flammarion, 2000 (citée sous la forme suivante : K et numéro de page ; ici : K 94).

(2) PE 46, K 94sq. 
de grandes entreprises industrielles, et où les banques étaient encore embryonnaires, pouvait passer pour la marque d'une conduite de vie approuvée par la morale, et même pour un impératif moral ? Parler ici d'un 'reflet' des conditions 'matérielles' dans la 'superstructure idéelle' n'aurait aucun sens » (3).

Si L'Éthique protestante s'ouvre sur des considérations statistiques, ce n'est certes pas seulement pour payer son tribut à la méthodologie de la sociologie naissante, qui émergeait à peine de la Nationalökonomie et était encore loin de s'être émancipée du rôle que jouent les statistiques dans l'École historique, représentée entre autres par Bruno Hildebrand et Karl Knies. En procédant ainsi, Weber montre en fait que les données statistiques laissent entier le problème du rapport de causalité entre protestantisme et capitalisme. Incontestablement, dans les États européens où les deux confessions, catholique et protestante, sont présentes la participation des protestants au capital ainsi que leur représentation au niveau "des chefs d'entreprise ainsi que des couches supérieures qualifiées de la main-d'œuvre, notamment, dans les entreprises modernes, du personnel technique ou commercial ou hautement qualifié » (4) sont proportionnellement plus fortes, mais cette relation ne se laisse expliquer entièrement ni par des raisons économiques, ni, à l'inverse, par le fait que le protestantisme serait plus «moderne» que le catholicisme. Du même coup L'Éthique protestante s'engage sur un terrain inexploré où elle ne va pouvoir compter que sur elle-même pour tenter d'élucider l' "affinité » troublante entre protestantisme et capitalisme.

Dans sa «Remarque préliminaire» aux Gesammelte Aufsätze zur Religionssoziologie Weber reconnaîtra que L'Éthique protestante n'éclaire, ce faisant, qu'un aspect des liens de causalité entre la religion et l'économie. À propos de l'hypothèse selon laquelle la relation entre protestantisme et capitalisme ne s'établit pas - et en tout cas ni entièrement ni prioritairement - dans l'ordre des causalités matérielles mais entre une éthique et un esprit, il admettra même dans une lettre à Rickert datée du 2 avril 1905 que son étude constitue " une sorte de reconstruction 'spiritualiste' de l'économie moderne ». Ce qu'elle entreprend, c'est - au sens de Rickert justement - une Kulturwissenschaft du capitalisme.

Mais il n'en néglige pas pour autant les conditions matérielles qui ont contribué à ce qu'une éthique religieuse ait des effets concrets, voire même les ont produits. Il ne défend nullement l'idée que les conceptions religieuses seraient indépendantes des conditions sociales dans lesquelles elles se développent. Dans L'Éthique religieuse des grandes religions mondiales, dès l'introduction, il rappellera au contraire que dans les religions du Salut la qualité des couches porteuses - bourgeois, paysans ou nobles guerriers - a été décisive pour la définition de la notion même de Salut (5). Dans L'Éthique protestante déjà il note qu'il n'y a certes pas de relation nécessaire entre la forme capitaliste de l'économie et 'l'esprit capitaliste' (6), mais que deux facteurs matériels n’en ont pas moins été déterminants :

(3) PE 63, K 123.

(4) PE 29, K 71

(5) «Die Wirtschaftsethik der Weltreligionen » in Gesammelte Aufsätze zur Religionssoziologie, Tübingen, J.C.B. Mohr, 1920, 9. Auflage 1988 - citée sous la forme suivante : WE et numéro de page; ici : WE 251.

(6) «Il est vrai qu'on observe généralement un rapport d' 'adéquation' entre la forme 'capitaliste' d'une entreprise et l'esprit dans lequel elle est dirigée, mais cette interdépendance n'est pas une 'loi' nécessaire. [...] En soi ces deux phénomènes peuvent parfaitement être dissociés » (PE 56, K 109). 
la rationalisation de la production et la mutation du statut des paysans, contraints à devenir des travailleurs salariés (7).

À la fin de son étude Weber formule le vœu que l'éclairage sous lequel il a placé la relation entre protestantisme et capitalisme ne soit pas à son tour considéré comme exclusif :

«[Dans le présent travail, nous avons d'abord tenté de ramener l'influence du protestantisme ascétique et ses modalités aux motivations dont ils étaient le produit en nous concentrant sur un seul point, même s'il s'agissait d'un point capital.] Mais il faudrait aussi montrer comment l'évolution et la spécificité de l'ascèse protestante ont de leur côté été influencées par l'ensemble des conditions culturelles, sociales, notamment les conditions économiques » (8).

Pour Weber, ainsi que le résume Wolfgang Schluchter, «il n'existe pas une explication exclusivement motivationnelle, ni non plus une explication exclusivement institutionnelle, ni une explication par les seules superstructures, ni une explication par la seule infrastructure, mais bien les deux à la fois » (9). "Toutes deux sont également possibles », déclare Weber lui-même à la fin de L'Éthique protestante, mais, ajoute-t-il, «l'une et l'autre, si elles ont l'ambition d'être le dernier mot d'une recherche et non un travail préparatoire, servent également peu la vérité historique » (10).

L'Éthique religieuse des grandes religions mondiales se consacrera expressément, à partir de 1911, à l'éclairage inverse et complémentaire de la religion par les conditions économiques et sociales. Dans la remarque préliminaire des Gesammelte Aufsätze zur Religionssoziologie, Weber résume ainsi la complémentarité entre ces deux entreprises :

«En tête du recueil figurent deux articles assez anciens (11) qui tentent d'aborder à partir d'un point particulier l'aspect du problème qui est le plus difficile à saisir : le rôle déterminant qu'ont joué certains contenus de croyance religieux dans l'émergence d'une 'mentalité économique', de l' 'ethos' d'une forme économique ; l'exemple choisi ici sera celui du lien entre l' ethos économique moderne et l'éthique rationnelle du protestantisme ascétique. On n'abordera donc dans un premier temps qu'un seul aspect de la relation causale qui nous occupe. Les études ultérieurement consacrées à 'l'éthique économique des religions mondiales', en donnant une vision globale des relations entre les religions des principales civilisations et l'économie et la stratification sociale du monde qui les entoure, tentent d'analyser les deux relations causales [...] » (12)

Car Weber tient non seulement pour insensée la thèse selon laquelle « l'histoire ne serait qu'une succession de prises de position et de luttes religieuses de l'humanité »(13), mais, plus précisément, il juge inapproprié de chercher à établir «la

(7) PE 57, K 113.

(8) PE 189sq, K 303. La phrase que nous mettons entre crochets est, significativement, un ajout de 1920, qui renforce les précautions déjà tout à fait explicites présentes dans la version de 1904-1905. Et pour faire bon poids Weber ajoute encore une note sur un texte de von Below (Die Ursachen der Reformation, Fribourg, 1916), à propos duquel il regrette précisément qu'il "n'aborde pas ce problème ».

(9) Wolfgang Schluchter, Religion und Lebensführung, Francfort/M., Suhrkamp, 1991, p. 72.

(10) PE $189 s q$, K $303 s q$.

(11) Il s'agit des deux chapitres qui constituent L'Éthique protestante.

(12) PE 21, K 63.

(13) Gesammelte Aufsätze zur Wissenschaftslehre, Tübingen, J.C.B. Mohr, 1973 (4e édition) citée sous la forme suivante: WL et numéro de page ; ici : WL 294. 
signification causale 'du religieux' dans sa globalité pour 'la vie sociale' prise elle aussi dans sa globalité » (14). L'Éthique protestante ne défend nullement la «thèse absurdement doctrinaire que 'l'esprit capitaliste' [...] a $p u$ n'être qu'un épiphénomène de certaines influences exercées par la Réforme, ou même que le capitalisme comme système économique est un produit de la Réforme » (15); elle se propose « seulement d'établir si certaines influences religieuses ont contribué à infléchir qualitativement et à diffuser quantitativement cet 'esprit' [capitaliste] dans le monde entier, jusqu'à quel point elles l'ont fait et quels aspects concrets de la culture qui s'est développée sur la base du capitalisme procèdent de ces influences religieuses », donc d'élucider "la manière dont un courant religieux a influencé l'évolution de la civilisation matérielle et le sens général dans lequel cette influence s'est exercée » (16).

\section{La polyrythmie de la rationalisation}

Non seulement L'Éthique protestante ne traite pas de l'influence directe de la ou d'une religion sur la réalité matérielle, mais de l'influence d'une éthique sur un esprit, non seulement elle pose que ce n'est qu'en tant qu'éthique qu'une religion peut influer de quelque manière sur la vie pratique et entrer ainsi dans une relation causale avec des réalités extra-religieuses, mais, y compris sous cet aspect, l'influence qu'elle exerce n'est nullement directe et ne se laisse aucunement assimiler à une causalité du type de celle dont traitent les sciences naturelles. Du fait de l'extrême diversité et complexité, de " l'incroyable enchevêtrement d'influences réciproques entre les fondements matériels, les formes d'organisation sociales et politiques et le contenu spirituel $\gg(17)$, on ne peut a priori procéder à aucune assertion d'ordre général. Cela vaut, selon la Wissenschaftslehre, pour tous les phénomènes sociaux et culturels (18). Aussi la combinaison de l'approche motivationnelle et d'une approche économico-sociale ne vise-t-elle pas à atteindre une connaissance totale dépassant le point de vue partiel de l'idéalisme et celui tout aussi partiel du matérialisme mais bien plutôt, comme l'écrit Wolfgang Schluchter, « à identifier les points nodaux où le passage à d'autres séries de déterminations serait intéressant » (19). Si Weber, au début de la deuxième section du premier chapitre, consacrée à "L' 'esprit' du capitalisme », parle d'un " complexe de relations qui existent dans la réalité historique et à partir duquel nous forgeons une unité conceptuelle en l'abordant du point de vue de sa signification pour une culture donnée », c'est pour souligner aussitôt qu'une telle " totalité conceptuelle» n'est que celle d'un point de vue particulier - «dans la perspective qui nous

(14) WL 295

(15) PE 76, K 151.

(16) PE 77, K 152

(17) Ibid.

(18) WL 295.

(19) Wolfgang Schluchter, Religion und Lebensführung, op. cit., p. 72. 
intéresse ici »-, que la perspective choisie - celle de la signification culturelle (Kulturbedeutung) - n'est pas la seule possible et qu'à la différence des lois générales des sciences de la nature elle ne "compose » jamais qu'un "phénomène significatif dans sa spécificité individuelle » (20).

La tendance à réduire cette complexité procède de la domination du modèle des sciences naturelles mais aussi du triomphe du capitalisme dont la vision mécaniste du monde occulte les processus culturels qui ont contribué à son avènement. La «science culturelle» de l' « esprit» capitaliste vise donc en quelque sorte à sortir de l'ombre l'histoire refoulée du modèle culturel dominant. Contre ce capitalisme victorieux qui semble ne plus avoir besoin du soutien des motivations et reposer sur une «base mécanique » (21), Weber fait valoir qu'il n'en a pas toujours été ainsi :

«De nos jours, les conduites de vie n'entretiennent le plus souvent aucune relation avec des motivations religieuses ; et lorsqu'une telle relation existe, elle est habituellement négative, en Allemagne en tout cas. [...] Il ne saurait plus être question aujourd'hui d'établir un lien nécessaire entre [la] conduite de vie 'chrématiste' et une quelconque 'vision du monde' unifiée. Il [l'ordre économique capitaliste] n'a plus besoin, notamment, de recevoir l'aval de certaines puissances religieuses et considère que l'influence exercée par les normes de l'Église sur la vie économique, si tant est qu'elle soit encore perceptible, est une entrave au même titre que les réglementations étatiques. [...] Mais ces phénomènes sont propres à une époque où le capitalisme moderne, désormais victorieux, s'est émancipé de ses anciens piliers » (22).

Il importe donc de «se demander comment un lien s'est établi entre la faculté d'adaptation au capitalisme à l'époque où il est apparu et les facteurs religieux » (23).

$\mathrm{Au}$ lieu de postuler un lien de détermination direct L'Éthique protestante avance l'hypothèse que dans les différentes sphères de l'existence s'accomplissent des processus de rationalisation qui ont leur rythme propre et obéissent à chaque fois à leurs lois propres. L'histoire « du» rationalisme ne montre " en rien des progrès simultanés dans les différentes sphères de la vie » (24). À ce moment de sa démonstration, à la fin de la deuxième section du premier chapitre, Weber se réfère à nouveau de façon critique à Werner Sombart et met en doute le fait que la naissance du capitalisme soit le résultat d'un processus de rationalisation qui se serait emparé de la même façon de tous les domaines de la culture comme de l'activité matérielle. Il oppose à cette vision aussi simpliste que grandiose l'exemple du Droit, qui dans les pays où le rationalisme économique a progressé le plus rapidement, au premier chef l'Angleterre, est demeuré rétrograde, et le contre-exemple de la philosophie, qui dans des pays plutôt en retard sur le plan économique, comme c'était le cas des pays catholiques et romans, n'en a pas moins fait avancer avec le plus d'audace la cause du rationalisme. Bref, « on peut 'rationaliser' - ce principe simple, que l'on oublie souvent, devrait figurer en tête de toute étude sur le 'rationalisme' - la vie selon des points de vue très différents et en fonction d'objectifs

(20) PE 39, K 85sq.

(21) PE 188, K 301.

(22) PE $59 \& 61, \mathrm{~K} 115 \& 117$.

(23) PE 53, K 107.

(24) PE 65, K 125. 
très différents. Le 'rationalisme' est un concept historique qui enferme en lui un monde d'oppositions » (25).

Dans ces conditions la relation entre ces deux processus de rationalisation que sont d'une part l'éthique protestante et d'autre part « l'esprit » capitaliste ne saurait être qualifiée que «de rapport d'adéquation »(Adäquanz-Verhältnis) ou encore d' " affinité élective ». Au lieu de postuler un lien de causalité, il convient plutôt de rechercher «s'il existe quelques 'affinités électives' entre certaines formes de foi religieuse et l'éthique du métier, et en quoi elles consistent» (26). Entre les institutions et les motivations également les rapports ne prennent la forme que d'une correspondance, d'une " adéquation » : «Il est vrai qu' on observe généralement un rapport d' 'adéquation' entre la forme 'capitaliste' d'une entreprise et l'esprit dans lequel elle est dirigée, mais cette interdépendance n'est pas une 'loi' nécessaire » (27).

À maintes reprises le relevé des «affinités électives » prend dans L'Éthique protestante la forme d'un simple constat d'analogies qui restent de pures analogies parce que Weber se garde de toute assertion généralisante risquant de trahir la complexité des enchevêtrements de déterminations qu'elles recouvrent. Lorsqu'il constate au début de son étude que les statistiques attestent le caractère majoritairement protestant du capital et de l'entreprise et, qu'à l'inverse, il existe une inclination manifeste des pays économiquement développés en faveur de la Réforme, ce ne sont là à ses yeux que la pure constatation de coïncidences dont on peut tout au plus inférer l'existence d' « affinités électives » mais certainement pas une relation de cause à effet. À la lecture de L'Éthique protestante on ne cesse d'ailleurs d'être frappé par le fait qu'elle va d'une "constatation frappante » (Auffälligkeit) à une autre, de l'observation d'une "adéquation » manifeste à une autre. Ainsi au milieu de la section sur «L' 'esprit' du capitalisme » :

«Si nous employons malgré tout - à titre provisoire - l'expression 'esprit du capitalisme (moderne)' pour désigner la mentalité qui vise, de manière systématique et rationnelle, par le biais d'un métier, un gain légitime [...], cela tient à une raison historique : c'est dans l'entreprise capitaliste moderne que cette mentalité a trouvé sa forme la plus adéquate, et l'entreprise capitaliste, de son côté, a trouvé en elle le moteur spirituel le plus adéquat » (28).

La «raison historique» (historischer Grund) ne peut certes pas dans ce passage être tenue pour une fondation de la relation constatée. À la fin de la première section on pouvait déjà lire : «Il suffira de rappeler la conjonction encore plus éclatante encore entre une réglementation religieuse de la vie et un sens des affaires très développé chez un grand nombre de sectes dont l' 'indifférence à la vie' est devenue aussi proverbiale que leur richesse, en particulier chez les quakers et les mennonites » (29). Du reste, à cet endroit, Weber fait en quelque sorte son autocritique en déclarant en conclusion : 
« Notre tâche devra précisément consister à formuler ce dont nous avons confusément l'intuition aussi clairement que l'inépuisable multiplicité inhérente à tout phénomène historique le permet» (30).

Pour tout à la fois rendre compte de «l'inépuisable multiplicité » et dépasser les «vagues généralités » une méthodologie était nécessaire, qui prend naissance dans L'Éthique protestante et s'y cristallise dans le concept d'idéaltype. Weber promeut ce concept en deux endroits décisifs de son étude : d'une part dans la section sur 'l'esprit' du capitalisme, où il s'agit de distinguer de tous ses prédécesseurs et précurseurs l'entrepreneur capitaliste nouveau pour qui l'accomplissement du métier est la seule source de satisfaction, d'autre part au début du deuxième chapitre, lorsqu'il s'agit de dégager la caractéristique générale des représentations religieuses qui ont pu motiver la conduite de vie spécifique du protestantisme ascétique :

«La seule manière possible de procéder est de présenter ces idées religieuses sous la forme d'une compilation cohérente et 'idéaltypique' qui ne s'est rencontrée que rarement dans la réalité historique. En raison même de l'impossibilité de tracer des frontières bien délimitées dans la réalité historique, nous ne pouvons espérer saisir leurs effets spécifiques qu'en étudiant leurs formes les plus conséquentes » (31).

C'est dans ces deux passages essentiels que prend corps l'hypothèse méthodologique sur laquelle repose la "science culturelle» qu'inaugure L'Éthique protestante : à supposer qu'il y ait bien une détermination de nature causale des processus historiques objectifs par les représentations subjectives, seule une « construction » ou, comme dit Weber, une «composition » idéal-typique peut en rendre compte.

Et cette hypothèse produit une sorte de coup de théâtre : la validité réelle de l'idéaltype va résulter du fait qu'il va débusquer une conséquence, non point certes au sens d'une relation de cause à effet entre la religion et la réalité économique mais au sens allemand de Konsequenz, au sens d'une logique interne impitoyable de l'éthos induit par le protestantisme et des comportements qu'il motive dans l'activité pratique des individus. C'est cette «conséquence » qui constitue la « spécificité » (Eigenart) de l'éthique protestante, et plus précisément puritaine et, par là-même, la spécificité du rapport «causal» entre protestantisme et capitalisme.

Parler de « religion du capitalisme » n'est en ce sens nullement une simple métaphore. En tant qu'éthique la religion joue bien « un rôle constitutif » (32) pour l'esprit du capitalisme moderne. Le comportement qui donne consistance à l' " affinité élective », c'est, dans le calvinisme, la recherche par l'individu croyant d'une confirmation (Bewährung), " une impulsion de contrôle méthodique de son état de grâce dans sa conduite » qui va jusqu'à l'ascèse et représente une « rationalisation de la conduite au sein du monde », « une mise en forme rationnelle de toute l'existence, conforme à la volonté de Dieu » (33). Ce sont donc, comme l'écrit Weber dans L'Éthique économique

(30) PE 38, K 85.

(31) PE 117, K 157. C'est Weber qui souligne «en raison », répondant ainsi à l'insuffisance de « raison historique ".

(32) PE 45, K 93.

(33) PE 164, K 250. 
des grandes religions mondiales, «les incitations pratiques à l'action fondées dans les aspects pragmatiques et psychologiques des religions qui entrent en ligne de compte »; lorsqu'on s'efforce d'établir la relation entre une religion, quelle qu'elle soit, et une réalité économique, «il ne peut s'agir que d'une tentative pour décortiquer les éléments déterminants de la conduite de vie des couches sociales qui ont influencé de la façon la plus forte l'éthique pratique de la religion considérée » (34).

\section{La multiplicité des rationalités : ni irrationalisme, ni relativisme}

Les religions mettent en œuvre des processus de rationalisation qui leur sont spécifiques. Il y a déjà une rationalité intrinsèque dans les promesses et les pratiques charismatiques des magiciens et il y en a une, a fortiori, dans les religions du Salut, qui entendent répondre à la question de la théodicée par une interprétation globale du monde (35). Ce que Weber entend par rationalité, et par rationalisation, ne saurait donc se réduire aux progrès d'une raison triomphant de l'irrationalisme - en particulier religieux. Du même coup on doit reconnaître aux religions d'avoir, selon leur logique propre, pris une part active dans la rationalisation du monde et des conduites. Si la thèse défendue par L'Éthique protestante contient une provocation, celle-ci réside dans la convergence, la collusion, "l'affinité » au premier chef rien moins qu'évidente, entre la rationalisation du monde économique mise en œuvre par le capitalisme et les motivations d'ordre apparemment irrationnel de l'éthique du métier. Mais sans doute faut-il aussi admettre qu'au sens wébérien de « rationalisation » le capitalisme développe une rationalité et une logique propres qui ne sont ni plus ni moins rationnelles, ou irrationnelles, que d'autres comportements pratiques et ne doivent en tout cas pas être tenues pour le triomphe de «la» rationalité sur l'irrationalité.

Sans aucun doute « du point de vue purement eudémoniste de l'intérêt personnel », la vocation exclusive au métier peut apparaître comme un comportement complètement irrationnel (36). Mais à une critique de Lujo Brentano Weber objecte que :

«l''irrationnel' n'est jamais une chose en soi, mais dépend d'un point de vue 'rationnel' déterminé. Pour celui qui est irréligieux, toute conduite de vie religieuse est irrationnelle, de même pour l'hédoniste toute conduite de vie ascétique est 'irrationnelle', quand bien même elle constituerait, à l'aune de sa valeur ultime, une 'rationalisation'. Si cet essai poursuit un but, c'est bien de contribuer à révéler la multiplicité de ce concept en apparence univoque du 'rationnel' » (37).

Le capitalisme moderne se distingue certainement par une forme de rationalité qui tranche avec les formes antérieures d'organisation de l'activité en vue de la

(34) WE $238 s q$.

(35) WE 244 sq.

(36) PE 66, K 126.

(37) PE $84 s q$, K 92 (traduction modifiée). 
recherche du gain. De ce (son) point de vue, ces dernières sont donc « irrationnelles ». De la même façon la religion est rejetée dans l'irrationnel lorsque la forme de « rationalisation » qu'elle a représentée devient en quelque sorte superflue. Et c'est notamment ce qui se passe dans le cas du capitalisme qui croit désormais pouvoir s'en tenir à l'autosuffisance de sa vision mécaniste du monde.

Or, la rationalité du protestantisme ascétique, la rationalisation qu'il a mise en œuvre, a consisté à parachever le processus de désenchantement du monde qu'avait engagé le judaïsme, à promouvoir une vision du monde délivrée de toute magie et à imposer une conduite de vie "rationalisée » à l'extrême en vertu de principes religieux. Ce fondement religieux est fourni par la doctrine calviniste de la prédestination et par son principe dogmatique "finitum non capax infinitum", c'est-à-dire que Dieu est un deus absconditus dont les intentions sont insondables. L'effet de ce dogme a été d'exclure «l'accessibilité d'un sens du monde pour la compréhension humaine [et de mettre fin] par là-même à toute problématique de cet ordre » (38). Par là il faut entendre au premier chef la théodicée comme tentative pour apporter une réponse rationnelle au problème du Mal et de la souffrance. Une forme de rationalité intrinsèquement religieuse, inspirant une conduite de vie éthique systématique, s'est en quelque sorte imposée contre une autre option rationnelle, celle que représentait la théodicée - qui constitue, comme on sait, un pont entre la théologie et la philosophie. La conséquence fut que seul le monde d'ici-bas offrait au croyant la possibilité de s'assurer, par la Bewährung, de sa justification. C'est pourquoi, pour résumer par un raccourci la thèse de Weber, une «affinité élective » (et en l'occurrence au sens fort de l'élection) s'est établie entre deux ordres de rationalisation a priori indépendants l'un de l'autre - une rationalité intrinsèquement religieuse et une rationalisation propre à l'existence dans le monde.

Peut-on aller plus loin et démontrer que cette « rencontre » était de quelque manière " nécessaire » ? C'est certainement tout l'enjeu de la réflexion de Weber, mais c'est aussi une question à laquelle il ne répond pas. Plutôt qu'une réponse à la question de la causalité, dont part L'Éthique protestante, il met en avant l'exigence, déjà citée, de reconnaître la pluralité des rationalités : "On peut 'rationaliser' la vie selon des points de vue très différents et en fonction d'objectifs très différents »; et dans la version de 1920 il ajoute précisément l'incise : «Ce principe simple, que l'on oublie souvent, devrait figurer en tête de toute étude sur le 'rationalisme'». Du même coup la rencontre entre «l'éthique protestante» et «l'esprit du capitalisme » reste un phénomène à la fois exemplaire et singulier - «ideal-typique » dans le meilleur des cas; par quoi il faut entendre : uniquement parce qu'il établit une nouvelle approche méthodologique dont le propre est de n'être transposable qu'en tant qu'exigence du respect de la singularité de l' «affinité élective » qu'elle débusque.

Le rationalisme peut «signifier des choses très différentes » (39). Non seulement la rationalité en fonction des fins (Zweckrationalität) est une chose complètement différente de la rationalité d'un système ou d'une vision du monde, mais, comme le montre L'Éthique économique des grandes religions mondiales en

(38) WE 573

(39) WE 265. 
étendant son champ d'investigation aux religions et aux éthiques religieuses extraoccidentales, la «rationalité » du confucianisme est foncièrement d'une autre nature que le rationalisme occidental. C'est pourquoi Weber a toujours souligné que L'Éthique protestante n'avait traité, avec ce dernier, que d'une évolution particulière et spécifique. En revanche, il revendique le mérite d'avoir proposé "une contribution à la typologie et à la sociologie du rationalisme en tant que tel » (40).

L'acquis en quelque sorte négatif de L'Éthique protestante consiste à avoir montré, à partir de l'évolution occidentale, «que l'histoire du rationalisme n'implique en rien des progrès simultanés dans les différentes sphères de la vie » (41). Ce qu'il fallait avant tout démontrer (et si ce n'était pas cela, c'est en tout cas la provocation théorique que Weber a léguée), c'est que l'esprit capitaliste n'est en aucun cas le produit, voire la conséquence nécessaire d'un mouvement général de rationalisation, d'un Progrès vers la Rationalité. La portée de cette démonstration va au-delà du refus du schéma d'interprétation base/superstructure. Car Marx avait bien souligné dans l'Introduction à la Critique de l'Économie politique que «l'énorme superstructure » se transforme plus ou moins rapidement que l'infrastructure économique et sociale. Mais il restait convaincu que malgré les décalages entre les rythmes d'évolution, voire même en dépit des contradictions qu'ils induisent et qui constituent justement la dynamique dialectique, et même révolutionnaire, de l'histoire, la tendance générale consistait dans l'affirmation du capitalisme qui s'emparait peu à peu de tous les secteurs du mode de production et que là où cette tendance toucherait à son terme elle basculerait inévitablement dans le socialisme. Marx, à cet égard, était l'héritier de la foi de l'Aufklärung en une unité et en un sens de la Raison dans l'histoire. Weber au contraire récuse d'emblée, dans L'Éthique protestante, l'hypothèse que «le développement de 'l'esprit capitaliste' doive être simplement considéré comme un phénomène spécifique au sein de l'évolution générale du rationalisme » et qu'il ne faudrait tenir compte du protestantisme que « dans la mesure où il aurait été le 'fruit précoce' de conceptions purement rationalistes de l'existence » (42). Il montre au contraire que dans l'évolution historique ainsi «idéalisée » des moments irrationnels ont joué un rôle tout autant que des moments rationnels. L'approche de Weber vise plus à relever les décalages qu'à produire une vision d'ensemble confirmant la croyance en « une » rationalité dont l'avènement, en dépit d'aléas, serait inéluctable. C'est notamment le cas, on l'a noté plus haut, lorsqu'il souligne que des pays plutôt en retard sur le plan économique, comme c'était le cas des pays catholiques, n'en ont pas moins fait avancer de façon décisive la cause du rationalisme. On peut même résumer l'intention, et en tout cas l'acquis, de L'Éthique protestante en disant qu'elle s'inscrit globalement en faux contre l'idée que « la relation de l'homme à son 'métier' comme à un devoir au sens où le requiert le capitalisme » (43) puisse être assimilée à un processus de sécularisation. Dans L'Éthique protestante Weber démontre exactement le contraire, à savoir qu'une rationalisation extrême du comportement procède de motifs religieux «irrationnels", ou, plus exactement, que ce n'est pas «le » rationalisme d'une Raison sûre d'elle-même qui a porté la

(40) WE 537.

(41) PE 65, K 125.

(42) PE 64, K 124.

(43) PE 65, K 125 (traduction modifiée). 
téléologie de la modernité mais, pour partie au moins, une rationalité intrinsèquement religieuse.

On imagine sans peine l'extrapolation de l'acquis de L'Éthique protestante. À l'époque Weber n'avait encore développé ni une sociologie des formes sociales de domination, ni une sociologie du droit, sans parler d'autres domaines culturels. Autant de domaines d'application, autant de rationalités (44). Et dans la mesure même où aucune de ces rationalités ne fournit en principe l'aune à laquelle évaluer les autres, elles se trouvent dans une situation de concurrence, sinon de conflit, depuis que le désenchantement du monde a provoqué leur différenciation et leur autonomisation. Comme le montre L'Éthique protestante, ce qui caractérise « l'esprit capitaliste » moderne est qu'il n'a précisément plus aucun besoin d' " un lien nécessaire entre [la] conduite de vie 'chrématiste' et une quelconque 'vision du monde' unifiée (45). L'évolution même de la rationalisation dissout paradoxalement l'exigence de liens nécessaires entre les ordres de valeurs et par conséquent aussi entre les deux ordres d'évolution - matérielle et spirituelle - dont L'Éthique protestante entendait élucider la relation. La provocation à laquelle conduit la rigueur méthodologique de L'Éthique protestante est que « la » rationalisation émancipe et autonomise les rationalités. Ainsi, l'esthétique et la religion n'ont entretenu de liens consubstantiels qu'aussi longtemps que la religion est demeurée magique - musique comme moyen d'accéder à l'extase, danses rituelles, images et statues comme objets de culte, etc. La religion du Salut dévalorise en revanche, en même temps que tout ce qui est mondain, la signification de l'art. L'art et la religion entrent même directement en concurrence lorsque l'art propose une forme de salut intérieure au monde. Il en va par ailleurs de même pour l'érotisme. L'ascèse rationnelle du métier ne reconnaît en effet que le mariage obéissant à des règles rationnelles et poursuivant des buts rationnels (la procréation et l'éducation des enfants). L'érotisme et la sexualité entrent eux aussi en conflit avec la religion lorsque, comme l'art, ils offrent une rédemption du quotidien rationnel - et ce d'autant plus qu'à l'extrême du processus de rationalisation occidental l'érotisme apparaît comme l'ultime lien « reliant à la source naturelle de toute vie l'homme qui est désormais complètement sorti du cycle organique de l'ancienne existence du simple paysan » (46). Quant à la science elle-même, contre toute apparence, elle n'est qu'une sphère de valeurs parmi d'autres.

Cette idée d'une pluralité des rationalités sur laquelle débouche l'offensive méthodologique de L'Éthique protestante contredisait tout à la fois l'idéal de la

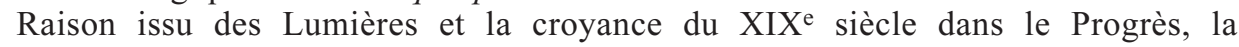
confiance encore entière dans le progrès d'une Raison embrassant toutes les sphères de l'existence. Il contredisait en particulier les philosophies de l'histoire qui se représentaient la marche de l'histoire universelle comme une progression éventuellement dramatique, mais inéluctable, vers la réconciliation et le dépassement des contradictions. Dans la troisième période de son évolution Weber a radicalisé sa conception de l'indépendance et du caractère à chaque fois " absolu » des différentes sphères de valeurs. Certes, dans la Wissenschaftslehre, le passage

(44) Cf. Schluchter, op. cit., p. 102 sq.

(45) PE $61, \mathrm{~K} 117$; c'est nous qui soulignons.

(46) WE 560. 
fameux de la "considération intermédiaire » où Weber parle de l'affrontement des dieux semble s'appliquer au présent, à une époque dans laquelle les «points de vue ultimes » qui déterminent l'action des hommes ont perdu le dénominateur commun que l'éthique religieuse a représenté des siècles durant. Mais il serait erroné de n'y voir qu'une critique de la civilisation contemporaine. Car l'éthique protestante avait déjà dû, elle aussi, s'imposer contre d'autres «points de vue » possibles et même historiquement bien ancrés. Et c'est précisément le problème de son triomphe historique sur ces autres points de vue que les essais sur L'Éthique protestante s'efforcent d'élucider. Dès cette étude Weber met donc en avant le conflit des valeurs, la lutte entre les points de vue et les rationalités, mais de façon tout aussi décidée, il souligne que sa conception ne débouche nullement sur un pur relativisme. Car non seulement une «conséquence» finit par l'emporter sur des conséquences moindres mais de tels conflits ne naissent que lorsque les «dieux », les sphères de valeurs qui affirment leur autonomie et se limitent mutuellement, font valoir une prétention à la validité absolue et imposent aux individus une exigence inconditionnelle à laquelle ils ne peuvent se soustraire et à laquelle ils doivent, d'une manière ou d'une autre, apporter une réponse qui les engage, positivement ou négativement.

\author{
Gérard RAULET \\ Université Paris-Sorbonne
}

Résumé

On simplifie souvent abusivement la théorie wébérienne de la rationalisation, notamment lorsqu'on la présente comme la théorie d'une "sécularisation » qui porterait l'évolution de "la rationalité occidentale». En récusant toute explication univoque, qu'elle soit causale ou motivationnelle, L'Éthique protestante montre que l'histoire du rationalisme n'implique en rien des progrès simultanés dans les différentes sphères de la vie. La démarche de Weber s'attache même plus à relever les décalages qu'à proposer une vision d'ensemble confirmant la croyance en " une » rationalité dont l'avènement, en dépit d'aléas, serait inéluctable. La provocation sur laquelle débouche cette conception est que "la " rationalisation émancipe et autonomise des rationalités.

Il en résulte deux conséquences, qui se complètent et se corrigent, et qui esquissent en quelque sorte une philosophie wébérienne de l'histoire. D'une part une relativisation du "rationnel » et de "l'irrationnel », en vertu de laquelle il y a autant de "rationalités " que d'ordres de valeurs. Dès L'Éthique protestante est donc présente l'idée d'un polythéisme des valeurs, d'une lutte entre les points de vue et les rationalités. Aucune de ces rationalités ne fournissant en principe l'aune à laquelle évaluer les autres, elles sont naturellement dans une situation de concurrence, voire de conflit.

Mais, d'autre part, Weber souligne que sa conception ne conduit nullement au relativisme. Car une "conséquence" finit par l'emporter. Dans le cas des rapports entre l'éthique protestante et le capitalisme, une "affinité élective " (en l'occurrence au sens fort de l'élection) s'est établie entre deux ordres de rationalisation a priori indépendants l'un de l'autre - une "rationalité " intrinsèquement religieuse et une rationalisation propre à l'existence dans le monde. 


\section{Abstract}

Weber's theory of rationalization is often improperly simplified, notably when it is presented as a theory of "secularization" supposedly driving the evolution of "Western rationality". Rejecting any univocal explanation, regarding either cause or motivation, The Protestant Ethic demonstrates that the history of rationalism does not imply simultaneous advances in the different spheres of life. On the contrary, Weber's approach tends more to stress the discrepancies rather than to propose a global vision confirming the belief in "one" rationality whose success advent would be inevitable despite some ups and downs. The provocative idea highlighted by such a conception is that "the" rationalization process liberates and grants autonomy to various rationalities.

This approach leads to two complementary and mutually corrective consequences that present, in a way, a weberian philosophy of history. On the one hand, it presents a relativization of the "rational" and the "irrational", according to which there are as many "rationalities" as there are spheres of values. As early of The Protestant Ethic we find the idea of a polytheism of values, of a struggle between points of views and rationalities. None of these rationalities offers, in principle, a norm according to which the others are measured. They find themselves in competition, even in conflict.

On the other hand, Weber stresses that his conception does not lead to relativism. Because one "consequence" eventually triumphs. In the case of the relationship between the Protestant ethic and capitalism, an "elective affinity" (here a strong sense of election) has developed between two apparently independent orders of rationalization - an intrinsically religious one and a rationalization related to worldly existence.

\section{Resumen}

Se simplifica a menudo abusivamente la teoría weberiana de la racionalización, especialmente cuando se la presenta como la teoría de una "secularización" de la cual la evolución de la "racionalidad occidental" sería portadora. Recusando toda explicación unívoca, sea ésta causal o motivacional, La Etica protestante muestra que la historia del racionalismo no implica de ninguna manera progresos simultáneos en las diferentes esferas de la vida. La perspectiva de Weber se propone dar cuenta de los desajustes más que proponer una visión de conjunto, que confirme la creencia en "una" racionalidad cuyo advenimiento sería ineluctable. La provocación en la que desemboca esta concepción es que "la" racionalización emancipa y autonomiza racionalidades.

Surgen entonces dos consecuencias, que se completan y se corrigen, y que esbozan de alguna manera una filosofía weberiana de la historia. Por un lado una relativización de lo "racional" y de lo "irracional", en virtud de la cual hay tantas "racionalidades" como órdenes de valores. Desde La Ética protestante se presenta entonces la idea de un politeísmo de los valores, de una lucha entre las perspectivas y las racionalidades. Dado que ninguna de estas racionalidades tiene la primacía para evaluar a las otras, éstas se hallan naturalmente en una situación de competencia, cuando no de conflicto.

Pero, por otra parte, Weber subraya que su concepción no conduce de ninguna manera al relativismo. Porque una "consecuencia" termina por primar. En el caso de las relaciones entre la ética protestante y el capitalismo, una "afinidad electiva" (aqui en el sentido fuerte de la elección) se ha establecido entre dos órdenes de racionalización a priori independientes el uno del otro, una "racionalidad" intrínsecamente religiosa y una racionalización propia de la existencia en el mundo. 
